\title{
The Phylogeny of Sleep Database: A New Resource for Sleep Scientists
}

\author{
Patrick McNamara*, ${ }^{*}$, Isabella Capellini ${ }^{2}$, Erica Harris ${ }^{1}$, Charles L. Nunn ${ }^{3,4}$, Robert A. Barton ${ }^{2}$ and \\ Brian Preston ${ }^{3}$
}

${ }^{1}$ VA New England Healthcare System and Boston University School of Medicine, Department of Neurology (127), 150
South Huntington Avenue, Boston, MA 02130, USA; ${ }^{2}$ Evolutionary Anthropology Research Group, Department of An-
thropology, Durham University, DH1 3HN, UK; ${ }^{3}$ Max Planck Institute for Evolutionary Anthropology, Deutscher Platz
No. 6, D-04103 Leipzig, Germany; ${ }^{4}$ Department of Integrative Biology, University of California, Berkeley, CA 94720,
USA

\begin{abstract}
We have constructed a database that describes the sleeping characteristics of 127 different mammalian species representing 46 families across 17 orders. The data were extracted from 178 separate references that were found using standardized search protocols, and for each study includes information on the time spent in REM and NREM sleep, sleep cycle length, the number of animals sampled, their sex and age, and reference citation. Importantly, we also coded nine laboratory condition scores as a way to control for the procedures that were used to collect the data. We created a website that contains the database (http://www.bu.edu/phylogeny/) from which others can both download the data and submit new results. This database is being used to address fundamental questions concerning the evolution of mammalian sleep; similar databases on other groups of organisms will enable sleep biologists to understand patterns of sleep at broader phylogenetic scales.
\end{abstract}

Keywords: Phylogeny, comparative analyses, REM, NREM, evolution.

\section{INTRODUCTION}

Evolutionary biologists recognize two main approaches to studying the function of behavioral traits such as REM (rapid eye movement) or NREM (non-rapid eye movement) sleep. One is to conduct experiments in model species that are aimed at determining the benefits and costs of a trait to individual fitness. The second approach is to use comparative methodologies to examine how the trait of interest evolved across different species, and identify the attributes that evolved in association with this trait. The comparative method has long been the cornerstone of evolutionary hypothesis-testing in biological sciences, for it is only through such inter-specific comparisons that we can make generalizations about how and why a trait has evolved (Brooks UOC 1991) [1] (Garland PZ 1994) [2] (Harvey OUP 1991) [3] (Ridley AB 1986) [4].

Here we describe the construction and content of a database that has been designed to enable the comparative study of mammalian sleep. The primary data included in this database are the total sleeping times, NREM and REM quotas, and sleep cycle lengths for a broad range of mammalian species. We begin with a brief consideration of the validity of sleep quotas as a measure worthy of study, and continue with descriptions of the database and its uses.

\section{Validity of Sleep Quotas in Comparative Analyses}

Inter-specific variation in time devoted to each of the two major mammalian sleep states (REM and NREM) is a physiologically meaningful index of sleep expression, sleep

*Address correspondence to this author at the VA New England Healthcare System and Boston University School of Medicine, Department of Neurology (127), 150 South Huntington Avenue, Boston, MA 02130, USA; E-mail: mcnamar@bu.edu function and sleep dysfunction. When deprived of the sleep amount they normally require, most of the animals whose sleep has been studied exhibit a sleep-rebound proportional to the amount of sleep lost, (Tobler ES 2000) [5] indicating that the amount of sleep, or of some specific intensity component of sleep reflected in 'amount of sleep', is physiologically obligatory. If sleep deprivation is prolonged, impaired physiological functions are manifest, ultimately causing death (as demonstrated in the rat, Rattus norvegicus) (Rechtschaffen Sleep 1989) [6].

Major human disorders of sleep manifest as changes in sleep amounts with either too little (insomnia) or too much (excessive daytime sleepiness) sleep (Carskadon ES 2006) [7]. People who experience too much or too little REM sleep, for example, tend to be at greater risk for ill-health. Increased REM sleep durations (relative to the population norm) are significantly associated with increased risk for various medical conditions and for mortality (Brabbins IJGP 1993) [8] (Dew PM 2003) [9] (Kripke PM 2003) [10] (Elgar AB 1988) [11]. The increased risk for these 'co-morbidities' remains even after adjusting for age, gender, mental illness and 'medical burden' or physical health status (Brabbins IJGP 1993) [8] (Dew PM 2003) [9] (Kripke PM 2003) [10].

Based on these considerations, the use of sleep quotas has long been a standard measure used in comparative tests, (Elgar AB 1988) [11] (Elgar AB 1990) [12] (Lesku AN 2006) [13] (Zepelin ES 1989) [14] and is the basis for construction of our database on sleep quotas.

\section{MATERIALS AND METHODOLOGY}

\section{Database Design and Construction}

In order to apply the comparative method, it is first necessary to compile relevant data for as many different species 
as possible. To achieve this aim, we conducted an exhaustive literature search dating back to 1953, the year REM sleep was discovered. We used the key terms REM, NREM, sleep architecture, EEG and total sleep time, to search bibliographic databases that cover publications in English, French, Russian, German, and Spanish. We also identified source material from previous data compilations.

A second basic requirement for comparative analyses is that the data from different sources must be comparable. For investigations of sleep, this can be achieved by either restricting analyses to studies that applied standardized assay procedures to the relevant age or sex of animals, or alternatively, by statistically controlling for procedural differences among studies. In each of these cases, however, we require details of the methodologies that different studies employed (see below). To obtain this crucial information, we gathered data only from the original source material and recorded information on laboratory procedures.

\section{Variation in Subject Animals}

We recorded the sex of individuals tested, and separated data for male and female subjects whenever possible. Similarly, we recorded the absolute age and age class (neonate, juvenile, adult) of experimental subjects when this was provided.

\section{Variations in Laboratory Procedures}

We systematically extracted information on the following laboratory conditions and experimental procedures from the original sources:

- Recording method (EEG). Whether sleep duration estimates were based on EEG recordings or not.

- Telemetry (yes or no). Whether sleep duration estimates were based on telemetric recordings in the wild.

- Recording time as reported in hours (categorical threestate variable). We categorized all studies according to recording times in the following way: $<12$ hours recording time, between 12 and $<24$ hours, and recording time equal to or longer than 24 hours.

- Diet (yes or no). Whether experimental animals were fed ad libitum or not.

- Normal lighting conditions (yes or no). If subjects were maintained under a 'natural' light condition of 12 hours light and 12 hours dark, or not (24 hours light or reversed light conditions).

- Ambient temperature (yes or no). Whether subjects were tested in an environment with temperatures within the natural range for the species under study.

- Adaptation (habituation; yes or no). Whether studies allowed the experimental animals to habituate to recording and laboratory conditions before data were collected.

- Restraint (yes or no). Whether or not subjects were restrained during sleep recording sessions.

- Behavioral observations (yes or no). Whether any sleeprelated behavioral observations were noted in the species under study. This could include any mention of sleep posture, eyes opening and closing, tail jerking, penile erection during REM, neck paralysis during REM, or related behaviors.

\section{Characteristics of Mammalian Sleep}

Our exhaustive search of the literature yielded 178 studies containing data for 127 species representing 46 families across 17 orders (see Table $\mathbf{1}$ ).

Table 1. Summary of Characteristics for Sleep Database

\begin{tabular}{|l|c|}
\hline Number of Papers & 178 \\
\hline Number of Species with Sleep Data & 127 \\
\hline Number of Families with Sleep Data & 46 \\
\hline Number of Orders with Sleep Data & 17 \\
\hline Number of Species with REM and NREM Sleep Data & 73 \\
\hline Number of Species with Sleep Cycle Data & 59 \\
\hline
\end{tabular}

Notes: REM = rapid eye movement; NREM = non-rapid eye movement.

From these data, we find that the 'average' mammalian adult spends 11.7 hours of each day asleep. $17 \%$ of this time is spent in REM sleep, with an average cycle length of 21 minutes between each episode of REM. A more accurate characterization of mammalian sleep may be in terms of its diversity. The amount of time devoted to sleep varies greatly, from three hours in the ass (sometimes incorrectly referenced as Equus asinus) (Ruckebusch CRSB 1963) [15] to 20 hours in the armadillo (Chaetophractus villosus) (Affani PBS 1972) [16]. The echidna (Tachyglossus aculeatus) does not appear to exhibit the classical EEG signs of REM sleep state (Allison AIB 1972) [17] (Siegel JN 1996) [18], in marked contrast to the duck-billed platypus (Ornithorhynchus anatinus), which spends $40 \%$ of its total sleeping time in REM sleep (Siegel Neuro 1999) [19]. Finally, sleep cycles may be as short as 6 minutes (in the chinchilla, Chinchilla laniger) (Van Twyver PB 1969) [20] or as long as 60 minutes (in the horse, Equus caballus) (Ruckebusch CRSB 1970) [21]. It is this remarkable variation in the nature of sleep across different species that makes the comparative analysis of mammalian sleep such an exciting and potentially fruitful endeavor.

\section{The Special Case of Sleep in Aquatic Mammals}

Members of three different orders that contain aquatic mammals - cetaceans (dolphins, porpoises, and whales), carnivores (seals, sea lions, and otters), and sirenians (manatees) have special sleep characteristics. Cetaceans and some carnivores typically engage in unihemispheric sleep (Mukhametov NL 1987) [22] (Lyamin AIB 2004) [23]. Pinnipeds within these groups are also able to sleep on land or in water, and there appears to be differences in sleeping quotas when they are asleep in water. Thus, to fully characterize sleep in these animals we require daily sleep quotas in both the right and left hemispheres on land and in the water. We have attempted to provide this information within our database, but we urge caution in using these data until a consensus emerges on how to best summarize sleep expression in marine or aquatic mammals.

\section{How to USE the Database}

The database is provided via a website funded by the National Institute of Mental Health (NIMH) (http://www.bu. edu/phylogeny/). This website provides background on hy- 
potheses for the function of sleep, details on how the database was constructed, and a portal to search the latest version of the database. A search algorithm has been developed that allows users to search the core Phylogeny of Sleep Database (http://www.bu.edu/phpbin/sleep/search/). A glossary of search terms is provided to enable visitors to extract key information from the database. Users can perform searches of the sleep dataset according to their own criteria (for example, selecting only those studies that utilized at least 24 hour recording periods), at which point an annotated reference and selected search information will be displayed. The full reference and record for any particular species can be displayed by clicking on this annotated reference. The data from searches can be downloaded, and searches can be saved as a file with values separated by commas (i.e., a .csv file, easily read by spreadsheet and word processing programs). Additionally, the upper right-hand corner of each page provides a 'Contact' button that allows users to send questions and comments about the database. Finally, we have established a user-friendly web-based mechanism that allows researchers to add new data on mammalian sleep durations to the database, following confirmation of data collection procedures according to the protocols we have developed. This link can be accessed from the search database page under 'Submit Study.'

\section{The Need for Phylogenetic Control}

Earlier studies of comparative sleep expression have called attention to the value of comparative analyses for improving our understanding of the function of sleep, most did not account for the similarity between species due to their common ancestry, an omission which can lead to erroneous conclusions (Harvey OUP 1991) [3] (Felsenstein AN 1985) [24] (Martins Evol 1991) [25] (Nunn EA 2001) [26]. Indeed, our recent analyses have detected a strong "phylogenetic signal' in mammalian sleep (Capellini Evol in press) [27]. In other words, closely related mammals are likely to share common sleep characteristics, creating inter-dependencies among the data points. Thus, the use of the analytical techniques that account for "phylogenetic non-independence" are required for analyzing sleep quotas extracted from the database (Nunn EA 2001) [26].

\section{DISCUSSION}

Here we showed how a "sleep informatics" approach can be used to build more comprehensive comparative databases on mammalian sleep. With such databases, it becomes possible to test functional hypotheses for the evolution of sleep and to do so in a way that controls for laboratory procedures. Our database is freely available over the World Wide Web, and by providing a means for others to submit data, we hope that the database will grow as this important field of research also grows. Similar databases can be developed for other groups of organisms, and by combining information at ever larger scales, we can begin to uncover the precursors of mammalian sleep while also investigating how sleep patterns differ in the major evolutionary radiations. We ask that users of the database cite this paper in any publications that result from searches of this database.

\section{ACKNOWLEDGEMENTS}

We would like to thank the following persons for assisting us with data extraction and data entry: Lena Giang, Pattie
Johnson, Mike Ma, and Sarah Varghese. We would like to thank Catherine Beauharnais, Nikkita Patel, Lana Ruvinskaya, and Alexandra Zaitsev for their assistance in translating articles.

This work was supported by NIMH grant number 1R01MH070415-01A1 to PM, RB and CN.

\section{REFERENCES}

[1] Brooks DR, McLennan DA. Phylogeny, ecology, and behavior: a research program in comparative biology. University of Chicago: Chicago, 1991.

[2] Garland T, Adolph SC. Why not to do two-species comparative studies: limitations on inferring adaptations. Physiol Zool 1994; 67: 797-828.

[3] Harvey PA, Pagel M. The comparative method in evolutionary biology. Oxford University Press: Oxford, 1991.

[4] Ridley M. The number of males in a primate troop. Anim Behav 1986; 34: 1848-1858.

[5] Tobler I. In: Kryger MH, Roth T, Dement WC, Eds. Principles and practices of sleep medicine. $3^{\text {rd }}$ ed. Philadelphia, Elsevier Saunders. 2000; 72-81.

[6] Rechtschaffen A, Bergmann BM. Sleep deprivation in the rat: an update of the 1989 paper. Sleep 2002; 25: 18-24.

[7] Carskadon MA, Dement WC. Normal human sleep: an overview. In: Kryger MH, Roth T, Dement WC, eds. Principles and practices of sleep medicine. $4^{\text {th }}$ ed. Philadelphia, Elsevier Saunders. 2006; 13-23.

[8] Brabbins CJ, Dewey ME, Copeland JRM, et al. Insomnia in the elderly: prevalence, gender differences and relationships with morbidity and mortality. Int J Geriatr Psychol 1993; 8: 473-480.

[9] Dew MA, Hoch CC, Buysse DJ, et al. Healthy older adults' sleep predicts all-cause mortality at 4 to 19 years of follow-up. Psychosom Med 2003; 65: 63-73.

[10] Kripke DF. Sleep and mortality. Psychosom Med 2003; 65: 74.

[11] Elgar MA, Pagel MD, Harvey PH. Sleep in mammals. Anim Behav 1988; 36: 1407-1419.

[12] Elgar MA, Pagel MD, Harvey PH. Sources of variation in mammalian sleep. Anim Behav 1990; 40: 991-995.

[13] Lesku JA, Roth TC, Amlaner CJ, et al. A phylogenetic analysis of sleep architecture in mammals: the integration of anatomy, physiology and ecology. Am Nat 2006; 168: 441-453.

[14] Zepelin H. Mammalian sleep. In: Kryger MH, Roth T, Dement WC, Eds. Principles and practices of sleep medicine. $2^{\text {nd }}$ ed. Philadelphia, Elsevier Saunders. 1989; 30-49.

[15] Ruckebusch Y. Etude EEG et comportamentale des alternantes veille-sommeil chez lane. CR Soc Biol 1963; 157: 840-844.

[16] Affani J. Observations on the sleep of some South American marsupials and edentates. Perspect Brain Sci 1972; 1: 21-23.

[17] Allison T, Van Twyver H, Goff WR. Electrophysiological studies of the echidna, Tachyglossus aculeatus. I. Waking and Sleep. Arch Ital Biol 1972; 110: 145-184.

[18] Siegel JM, Manger PR, Nienhuis R, et al. The echidna Tachyglossus aculeatus combines REM and non-REM aspects in a single sleep state: implications for the evolution of sleep. J Neurosci 1996; 16: 3500-3506.

[19] Siegel JM, Manger PR, Nienhuis R, et al. Sleep in the platypus. Neuroscience 1999; 91: 391-400.

[20] Van Twyver H. Sleep patterns in five rodent species. Physiol Behav 1969; 4: 901-905.

[21] Ruckebusch Y, Barbey P, Guillemot P. Les etats de sommeil chez le Cheval (Equus caballus). CR Soc Biol 1970; 31: 658-665.

[22] Mukhametov LM. Unihemispheric slow-wave sleep in the Amazonian dolphin, Inia geoffrensis. Neurosci Lett 1987; 79: 128-132.

[23] Lyamin OI, Mukhametov LM, Siegel JM. Relationship between sleep and eye state in cetaceans and pinnipeds. Arch Ital Biol 2004; 142: 557-568.

[24] Felsenstein J. Phylogenies and the comparative method. Am Nat 1985; 125: 1-15. 
[25] Martins E, Garland T. Phylogenetic analyses of the correlated evolution of continuous characters: a simulation study. Evolution 1991; 45: 534-557.
[26] Nunn CL, Barton RA. Comparative methods for studying primate adaptation and allometry. Evol Anthropol 2001; 10: 81-98.

[27] Capellini I, Barton RA, McNamara P, et al. A phylogenetic analysis of the ecology and evolution of mammalian sleep. Evol; in press. 